\title{
Non-pulmonary vein foci induced before and after pulmonary vein isolation in patients undergoing ablation therapy for paroxysmal atrial fibrillation: incidence and clinical outcome
}

\author{
Hui CHENG ${ }^{\S 1}$, Yin-yin DAI ${ }^{\S 2}$, Ru-hong JIANG ${ }^{1}$, Qiang LIU $^{1}$, Ya-xun SUN ${ }^{1}$, Jian-wei LIN ${ }^{1}$, \\ Zu-wen ZHANG ${ }^{1}$, Shi-quan CHEN $^{1}$, Jun ZHU ${ }^{1}$, Xia SHENG ${ }^{1}$, Chen-yang JIANG ${ }^{\dagger 1}$ \\ ( ${ }^{1}$ Department of Cardiology, Sir Run Run Shaw Hospital, School of Medicine, Zhejiang University, Hangzhou 310016, China) \\ ( ${ }^{2}$ Department of Cardiology, the First People's Hospital of Xiaoshan, Hangzhou 311200, China) \\ †E-mail: jiangchenyang@hotmail.com \\ Received May 27, 2014; Revision accepted Aug. 10, 2014; Crosschecked Sept. 24, 2014
}

\begin{abstract}
Objective: To evaluate the incidence and clinical outcome of adenosine triphosphate (ATP) plus isoproterenol (ISP)-induced non-pulmonary vein (PV) foci before and after circumferential PV isolation (CPVI) during index ablation in patients with paroxysmal atrial fibrillation (PAF). Methods: In 80 consecutive patients undergoing catheter ablation for drug-refractory, symptomatic PAF at our hospital from April 2010 to January 2011, atrial fibrillation (AF) was provoked with ATP $(20 \mathrm{mg})$ and ISP $(20 \mu \mathrm{g} / \mathrm{min})$ administration before and after CPVI. The spontaneous initiation of $A F$ was mapped and recorded. Results: Before ablation, $A F$ mostly originating from PVs (PV vs. non-PV, 36/70 vs. 3/70; $P<0.01$ ) was induced in 39 patients with sinus rhythm. CPVI significantly suppressed AF inducibility; however, more non-PV foci were provoked (post-CPVI vs. pre-CPVI, $13 / 76$ vs. 3/70; $P=0.016$ ). Patients with pre- and post-CPVI induced AF ( $n=49$ ) were divided according to non-PV foci being induced (group N, $n=17$ ) or not (group P, $n=32$ ). After mean (19.2 \pm 8.2$)$ months follow-up, $88.2 \%$ (15/17) and $65.6 \%$ (21/32) of patients in groups $N$ and $P$, respectively, were free from $\mathrm{AF}$ recurrence $(P=0.088)$. Conclusions: ATP+ISP administration effectively provokes non-PV foci, especially after CPVI in PAF patients. Although in this study difference did not achieve statistical significance, supplementary ablation targeting non-PV foci might benefit clinical outcome.
\end{abstract}

Key words: Atrial fibrillation, Non-pulmonary vein foci, Adenosine triphosphate doi:10.1631/jzus.B1400146 Document code: A CLC number: R541.7 $7^{+} 5$

\section{Introduction}

Pulmonary vein isolation (PVI) became the cornerstone catheter ablation procedure for paroxysmal atrial fibrillation (PAF) since pulmonary vein (PV) firing was reported as its major cause (Haïssaguerre et al., 1998). However, long-term outcome of PVI in PAF patients has been disappointing (Ganesan

\footnotetext{
* Corresponding author

$\S$ The two authors contributed equally to this work

(C) Zhejiang University and Springer-Verlag Berlin Heidelberg 2014
}

et al., 2013), and electrophysiological studies during repeat procedures revealed that besides PV reconnection, non-PV foci play an important role in $\mathrm{AF}$ recurrence (Chen et al., 1999; Sotomi et al., 2013). Non-PV foci mainly have been found in the superior vena cava (SVC), coronary sinus (CS), left atrial free wall, crista terminalis (CT), ligament of Marshall (LOM), and atrial septum (Shah et al., 2003). In this study, we evaluated the incidence and clinical outcome of non-PV foci induced by adenosine triphosphate (ATP) plus isoproterenol (ISP) administration before and after PVI during index ablation in PAF patients. 


\section{Materials and methods}

\subsection{Study population}

Eighty consecutive patients (52 males, (58.8 \pm 10.4) years) with drug-refractory, symptomatic PAF, who received catheter ablation procedure between April 2010 and January 2011 at our center, were enrolled in the study. Mean atrial fibrillation (AF) duration was $(5.2 \pm 5.5)$ years. PAF was defined as $\geq 2$ recurrent $\mathrm{AF}$ episodes spontaneously terminating within $7 \mathrm{~d}$. Exclusion criteria were: (1) contraindication to anticoagulation, ISP or ATP; (2) left atrial thrombus; and, (3) prior ablation in left atrium (LA).

\subsection{Electrophysiological study and ablation procedure}

Informed consent was obtained from each patient. All antiarrhythmic drugs except amiodarone were discontinued for $\geq 5$ half-lives before ablation. Transesophageal echocardiography was performed on the day before ablation to rule out left atrial thrombus. All procedures were performed under conscious sedation with fentanyl and midazolam.

CS catheter was placed between the left inferior pulmonary vein (LIPV) and mitral annulus to record the CS and LA. Immediately after successful transseptal puncture, intravenous heparin was given as a $5000 \mathrm{U}$ bolus followed by $1000 \mathrm{U} / \mathrm{h}$ during the remainder of the procedure. After selective PV angiography, multipolar circular (Lasso $^{\mathrm{TM}}$, Biosense Webster, Diamond Bar, CA, USA) and ablation (Navi-star ThermoCool, Biosense Webster) catheters were positioned at the right superior PV (RSPV) and left superior PV (LSPV) ostia, respectively, or into the inferior PVs if suspected as ectopic focus (Fig. 1).

Ten of the 80 patients were in $\mathrm{AF}$ at procedure onset, while the other 70 had sinus rhythm (SR). ATP $(20 \mathrm{mg})$ was rapidly injected intravenously during continuous infusion of ISP $(20 \mu \mathrm{g} / \mathrm{min})$ to induce $\mathrm{AF}$, and repeated if no $\mathrm{AF}$ was induced. The ectopic focus was localized according to the earliest atrial activity relative to reference electrogram or ectopic $\mathrm{P}$ wave onset.

Circumferential PV isolation (CPVI) was then performed, guided by the Lasso catheter and electroanatomic mapping system $\left(\mathrm{CARTO}^{\mathrm{TM}}\right.$, Biosense Webster). Radiofrequency energy was delivered at 5-10 mm from the $\mathrm{PV}$ ostia at $45^{\circ} \mathrm{C}$ (power: $25-35 \mathrm{~W}$; irrigated flow rate: $17-25 \mathrm{ml} / \mathrm{min}$ ). The endpoint was electrical isolation of all PVs with circular mapping catheter within circumferential lines showing no PV potential or presence of dissociated PV potential. The details of the techniques were described as before (Jiang et al., 2014).

In patients with SR after successful CPVI, ISP+ATP was administered again to provoke AF; non-PV areas, including $\mathrm{CS}, \mathrm{SVC}$, left atrial wall, LOM, right atrium (RA), and interatrial septum, were mapped. In patients who remained in AF after CPVI, empirical ablation was performed at sites with the shortest cycle length, the potential driver for $\mathrm{AF}$, ATP+ISP induction was repeated, and procedure endpoint was no AF induced by ISP+ATP (Fig. 2).

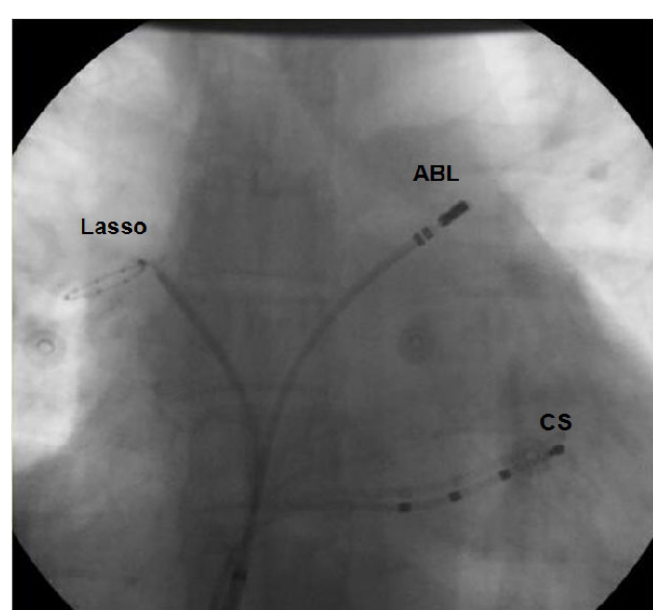

Fig. 1 Fluoroscopy showing catheter location during inducement

Lasso: circular mapping catheter; ABL: ablation catheter; CS: coronary sinus

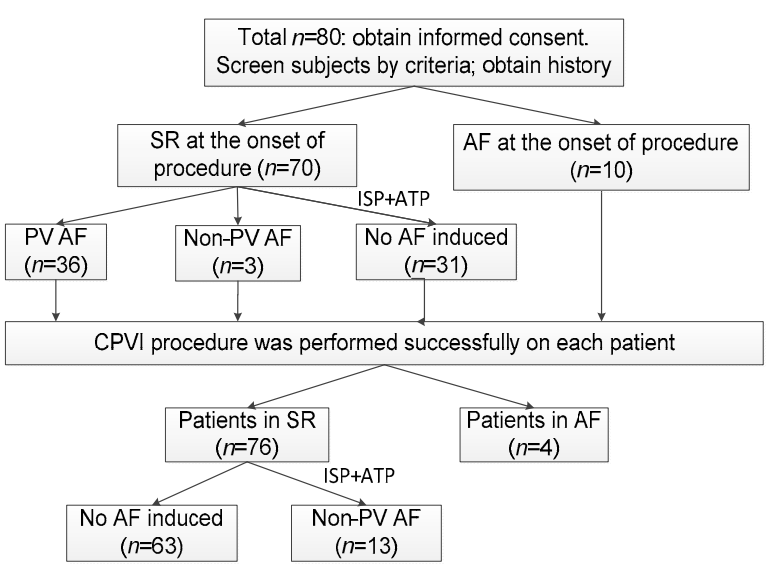

Fig. 2 Flowchart of study design and results 
Patients with induced AF were divided into two groups according to AF origin, either PV trigger(s) only (group P) or non-PV foci (group N).

\subsection{Follow-up}

Patients were followed up at 1, 3, 6, 12, and 24 months post-ablation. Warfarin was taken at least 3 months after the procedure. The target value of international normalized ratio (INR) was between 2 and 3. All antiarrhythmic drugs (AADs) were discontinued at three months post-procedure. Twelvelead electrocardiograph (ECG) and 24-h Holter ECG recordings were performed at each follow-up visit, and at any time symptoms were suggestive of arrhythmia. AF recurrence was defined as $>30 \mathrm{~s}$ of documented AF, atrial flutter, or atrial tachycardia. The first 3 months were taken as the blanking period.

\subsection{Statistical analysis}

All continuous variables are presented as mean \pm standard deviation (SD), and were analyzed with Student's $t$-test. Categorical variables are presented as numbers and percentage, and were compared with the chi-square test or Fisher's exact test. Kaplan-Meier survival curve and the log-rank test were used to describe and compare rates of freedom from recurrent AF. $P \leq 0.05$ was considered statistically significant. Statistical analyses were performed using SPSS Statistics 16.0 software.

\section{Results}

\subsection{Baseline characteristics}

Among the 80 patients studied, 49 patients had AF induced with ATP+ISP administration: 32 from PV foci (Group P) before and after CPVI, and 17 from non-PV foci (Group N). There were no significant baseline differences between the two groups (Table 1).

\subsection{Features of non-PV ectopic foci}

Before PV ablation, 70/80 patients were in SR, and AF was induced in 39 patients: 36 originating from PV (left PV 20, right PV 16), and 3 from non-PV foci. In these three patients with non-PV foci before CPVI, earliest atrial activities were recorded at proximal of CS catheter.

CPVI was successfully achieved in all patients. Four patients remained in AF rhythm after CPVI. Non-PV areas were mapped, AF cycle-length was measured, and ablation was performed at suspected AF origin sites. Three patients had AF terminated during ablation, including one at CS ostium and two at the left atrial anterior wall. One patient remained in AF despite additional ablation and received cardioversion.

Of 76 patients with SR post-CPVI, AF was induced in 13 by ATP, all originating from non-PV areas (13/76 vs. $3 / 70 ; P=0.016$, as compared with the incidence before CPVI; Fig. 3) including SVC (5/13), CS ostium (5/13), left atrial free wall (1/13), and unknown $(2 / 13)$. All areas located were successfully ablated and reconfirmed with ATP+ISP provocation (Figs. 4-6). Non-PV foci defined as unknown were difficult to locate in two patients: one only had a short AF episode induced and the other had sustained AF induction but could not be terminated by empirical ablation.

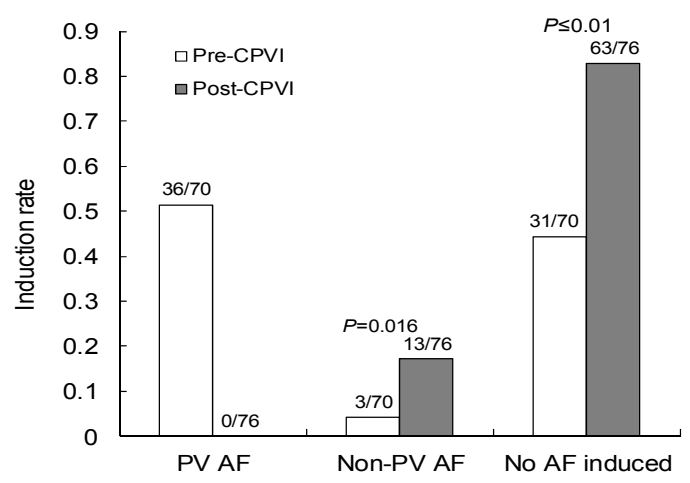

Fig. 3 Pre- and post-CPVI induction rates

Table 1 Baseline and characteristics

\begin{tabular}{|c|c|c|c|c|c|c|c|c|c|c|}
\hline \multirow[b]{2}{*}{ Group } & \multirow{2}{*}{$\begin{array}{l}\text { Age } \\
\text { (year) }\end{array}$} & \multirow{2}{*}{$\begin{array}{c}\text { AF } \\
\text { duration } \\
\text { (year) }\end{array}$} & \multirow{2}{*}{$\begin{array}{c}\text { BMI } \\
\left(\mathrm{kg} / \mathrm{m}^{2}\right)\end{array}$} & \multicolumn{5}{|c|}{ Number of subjects } & \multirow{2}{*}{$\begin{array}{l}\text { LAD } \\
(\mathrm{mm})\end{array}$} & \multirow{2}{*}{$\begin{array}{l}\text { LVEF } \\
(\%)\end{array}$} \\
\hline & & & & Male & HTN & CAD & Diabetes & MVD & & \\
\hline Total & .4 & $5.2 \pm 5.5$ & $24.1 \pm 3.0$ & $52(65 \%)$ & $36(45 \%)$ & $4(5 \%)$ & $2(2.5 \%)$ & $7(8 \%$ & 396 & $68.4 \pm 8.0$ \\
\hline Group P $(n=32)$ & $59.1 \pm 11.6$ & $4.8 \pm 4.8$ & $24.1 \pm 2.6$ & $21(66 \%)$ & $13(41 \%)$ & $2(6 \%)$ & $2(6 \%)$ & $2(6 \%)$ & $39.2 \pm 3.6$ & $68.4 \pm 8.1$ \\
\hline Group N ( $n=17)$ & $57.0 \pm 11.3$ & $6.7 \pm 8.1$ & $23.1 \pm 3.3$ & $11(65 \%)$ & $7(41 \%)$ & $0(0 \%)$ & $0(0 \%)$ & $2(18 \%)$ & $41.8 \pm 2.5$ & $68.1 \pm 8.9$ \\
\hline$P$ value & 0.507 & 0.176 & 0.116 & 1.000 & 1.000 & 0.541 & 0.541 & 0.634 & 0.220 & 0.832 \\
\hline
\end{tabular}

Data are expressed as mean $\pm \mathrm{SD}$ or number (percentage). BMI: body mass index; HTN: hypertension; CAD: coronary artery disease; MVD: mitral valve disease; LAD: left atrial dimension; LVEF: left ventricular ejection fraction 
(a)

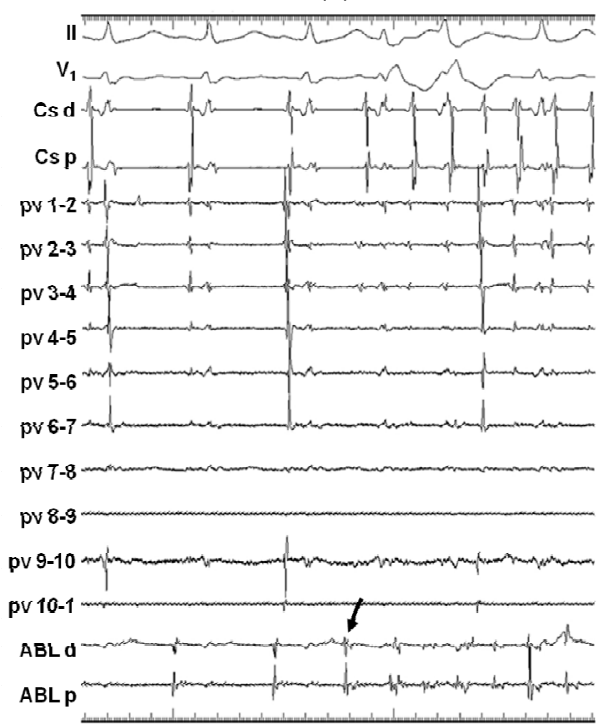

(b)

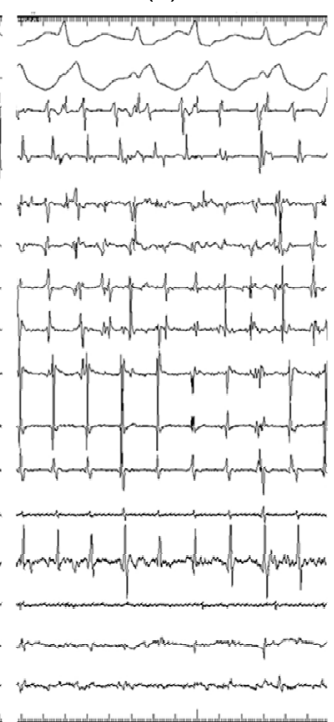

(c)

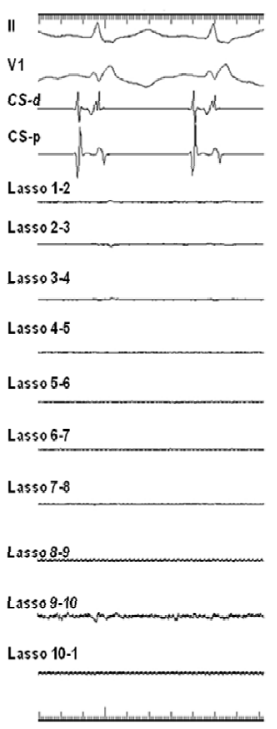

(d)
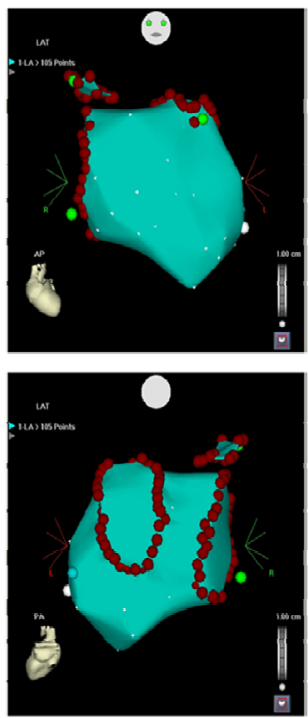

Fig. 4 Typical examples of firing and AF originating from SVC

(a) AF was induced after CPVI. Lasso catheter in LSPV; ABL catheter in RSPV. Arrow: far-field potential represented SVC recorded in RSPV was earliest. (b) Lasso catheter was positioned in SVC showing firing potentials with the shortest cycle-length; ABL catheter in RSPV. (c) Sinus rhythm restored after SVC isolation. (d) 3D electroanatomic map in CARTO system of LA in anterior and posterior views showing ablation lesions. Tracings correspond to ECG leads II and V1; CS-d, CS-p: distal and proximal pairs of CS catheter, respectively; PV 1-2 to PV 10-1 (Lasso 1-2 to Lasso 10-1): 10 bipolar pairs of the Lasso catheter; ABL-d, ABL-p: distal and proximal pairs of ablation catheter, respectively

(a)

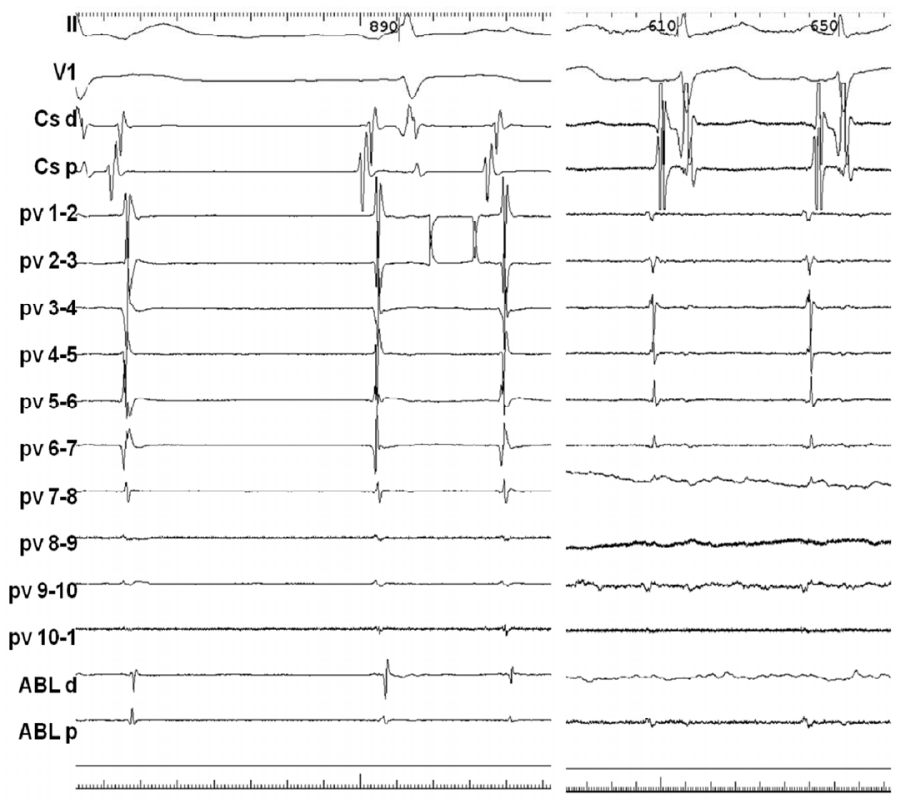

(c)

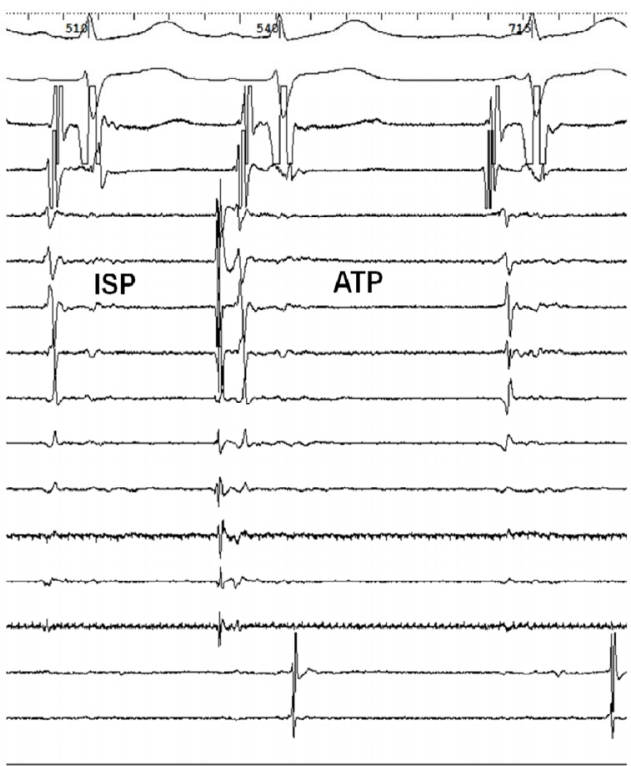

Fig. 5 Typical examples of firing and AF originating from CS

(a) AF was induced before CPVI. Lasso catheter in LSPV; ABL catheter in RSPV. CS-p showed the earliest ectopic beats. (b) After successful CPVI and CS ablation, AF stopped. (c) AF could not be induced by ATP+ISP. Lasso catheter in LSPV; ABL catheter in RSPV. Tracings correspond to ECG leads II and V1; CS-d, CS-p: distal and proximal pairs of CS catheter, respectively; PV 1-2 to PV 10-1: 10 bipolar pairs of the Lasso catheter; ABL-d, ABL-p: distal and proximal pairs of ablation catheter, respectively 
(a)

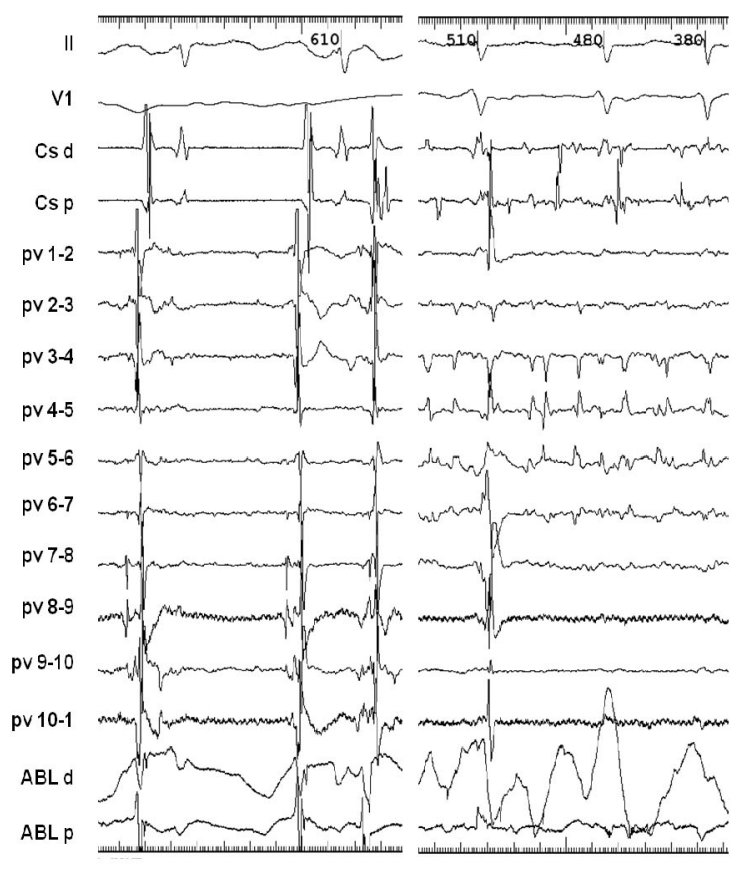

(c)

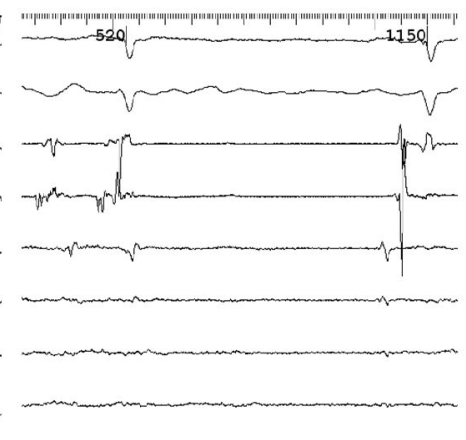

(d)
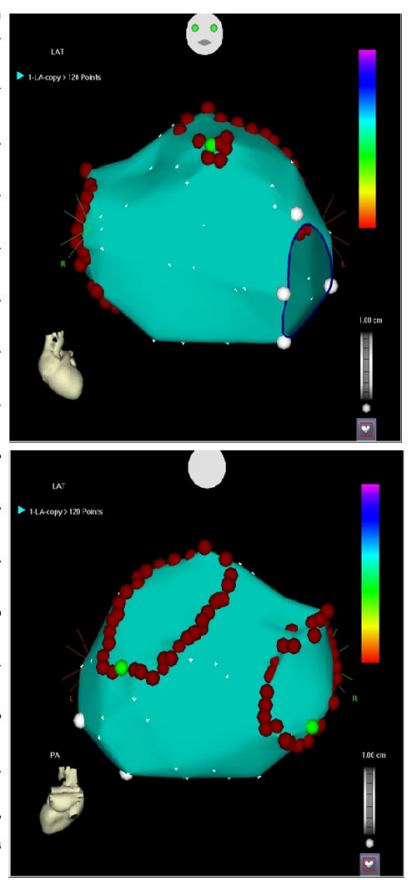

Fig. 6 Typical examples of firing and AF originating from left atrial anterior wall

(a) AF was induced during left PV ablation. Pulmonary vein potential reversal was not observed. Lasso catheter in LSPV; ABL catheter in the pulmonary vein antrum. (b) Lasso catheter in LSPV; AF persists after CPVI. (c) AF stopped during ablation of left atrial anterior wall. Lasso catheter in LSPV; ABL catheter in left atrial anterior wall. (d) 3D electroanatomic map in CARTO system of LA in anterior and posterior views showing ablation lesions. Tracings correspond to ECG leads II and V1; CS-d, CS-p: distal and proximal pairs of CS catheter, respectively; PV 1-2 to PV 10-1: 10 bipolar pairs of the Lasso catheter; ABL-d, ABL-p: distal and proximal pairs of ablation catheter, respectively

\subsection{Follow-up}

After a mean follow-up of (19.2 \pm 8.2$)$ months, $65.6 \%(21 / 32)$ and $88.2 \%(15 / 17)$ of patients were free from AF recurrence in groups $\mathrm{P}$ and $\mathrm{N}$, respectively, with difference not reaching statistical significance by log-rank test $(P=0.088$; Fig. 7).

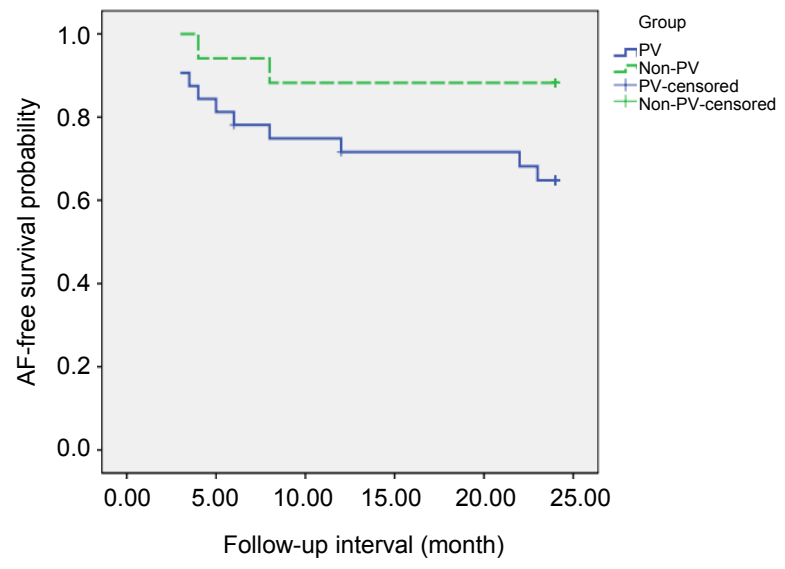

Fig. 7 Kaplan-Meier survival curves showing similar freedom-from-AF rates in the two groups

\section{Discussion}

\subsection{Main findings}

In this study, 3 patients had non-PV foci-initiated $\mathrm{AF}$ induction before CPVI, and 13 after CPVI. Although prior to $\mathrm{PV}$ isolation $\mathrm{PVs}$ played a predominant role in AF induction, PVI markedly suppressed PV rapid firing but enhanced inducibility of non-PV AF, albeit in a lower percentage of patients. With additional ablation, patients with non-PV foci had a numerically but not significantly lower clinical $\mathrm{AF}$ recurrence rate as compared to those with only PV foci.

\subsection{Incidence of AF before and after CPVI}

$\mathrm{AF}$ is a complex arrhythmia with multiple possible mechanisms (Allessie et al., 2001). The concept that the onset and maintenance of AF require both a trigger and a substrate was well accepted. Ectopic foci occurring in muscular sleeves of PV or vena caval junctions were considered as critical AF triggers 
(Haïssaguerre et al., 1998; Lin et al., 2003). Selfperpetuation process of AF including structural (atrial fibrosis, dilation, etc.) and electrical (shortening and dispersion of refractory periods) remodeling would be the substrate that sustains arrhythmia (Morillo et al., 1995; Wijffels et al., 1995; Goette et al., 1996).

Recent studies revealed a close relationship of the cardiac autonomic nervous system (CANS) with AF initiation (Nakagawa et al., 2009). Clinically, obvious fluctuation in autonomic tone could be recorded during continuous ECG recording before AF onset, especially in paroxysmal AF patients without structural heart disease (Zimmermann and Kalusche, 2001). Animal studies found that autonomic nerve stimulation can initiate AF (Patterson et al., 2005; Po et al., 2006) or facilitate conversion of stimulated premature depolarization from PVs into AF.

The aforementioned findings constitute the basis of our study using ISP and ATP to induce AF by simulating sympathetic and parasympathetic activation. ATP activates the autonomic nervous system (Lerman and Belardinelli, 1991); adenosine, the metabolite of ATP, activates $I_{\text {kAdo }}$ outward potassium ion channels and slows $I_{\mathrm{CaL}}$ reactivation, thereby hyperpolarizing the atria, and shortening action potential duration and effective refractory period (Workman et al., 1999). ISP raises intracellular calcium concentration, increasing heart rate and decreasing atrial refractory period. Besides the fluctuation in autonomic tone these drugs created, the direct pro-arrhythmic effect of them may increase the automaticity at PV and non-PV sites.

The findings described above could be supported by the marked suppression seen in PV-originated firing after CPVI. However, inducibility of non-PV $\mathrm{AF}$ was enhanced and non-PV foci were unmasked after PVs were isolated or irritated by substrate modification around PV ostia.

In the human heart, there are four major LA ganglionated plexi (GPs) located around the antrum of PVs, which are areas of high-density autonomic innervation. GPs receive input from the central autonomic nervous system, contain afferent and efferent neurons, and communicate with each other (Armour $e t$ al., 1997). Extra-PV foci may be irritated after the pathway between GPs and myocardial sleeves around
PV is blocked.

Besides autonomic innervation abundance around thoracic veins favoring ectopic beat origination, the myofascial architecture at the venoatrial junction is also the ideal substrate for reentry during AF initiation. Muscular discontinuities and abrupt changes in fiber orientation are found in more than $50 \%$ of PVs and PV-LA junction. Muscular sleeves around SVC (Zipes and Knope, 1972) and CS musculature can also be the source of atrial tachyarrhythmia (Morita et al., 2012), which might explain why SVC and CS were dominant sites of non-PV AF induction after CPVI.

\subsection{Outcome of patients with non-PV foci ablation}

In our study, with additional non-PV foci ablation during the index procedure, patients with non-PV foci had a numerically but not significantly lower clinical AF recurrence rate as compared to those with only PV foci. Inconsistent results in previous studies (Yamaguchi et al., 2010; Chang et al., 2013) might be secondary to differences at baseline, in AF type and provocation methods, and among others. As is the case for PV isolation procedure, supplementary ablation for some non-PV foci is an empirical therapy, and some non-PV firing may be an epiphenomenon of a real source located in the LA. More appropriately powered clinical studies are needed to evaluate the benefit of this strategy; by power calculation, 63 patients in each group would be needed to show a significant difference for the numerical difference observed between these two groups $(\alpha=0.05,1-\beta=$ $0.8)$.

\subsection{Limitations}

Firstly, a controlled study could not be designed because it is unethical to set up a group in which non-PV foci are induced but not ablated; however, this nonrandomized study is prospective. Secondly, the study was limited by the relatively small sample size. Thirdly, we only positioned catheters in the LSPV and RSPV because of limited transseptal access to the LA. Lastly, asymptomatic AF episodes might have been missed during follow-up even though ECG and 24-h Holter monitoring were performed on schedule. 


\section{Conclusions}

ATP+ISP administration effectively provokes non-PV foci, especially after CPVI in paroxysmal AF patients. Supplementary ablation targeting non-PV foci might benefit clinical outcome of patients with non-PV foci.

\section{Compliance with ethics guidelines}

Hui CHENG, Yin-yin DAI, Ru-hong JIANG, Qiang LIU, Ya-xun SUN, Jian-wei LIN, Zu-wen ZHANG, Shi-quan CHEN, Jun ZHU, Xia SHENG, and Chen-yang JIANG declare that they have no conflict of interest.

All procedures followed were in accordance with the ethical standards of the responsible committee on human experimentation (institutional and national) and with the Helsinki Declaration of 1975, as revised in 2008 (5). Informed consent was obtained from all subjects for being included in the study. Additional informed consent was obtained from all patients for which identifying information is included in this article.

\section{References}

Allessie, M.A., Boyden, P.A., Camm, A.J., et al., 2001. Pathophysiology and prevention of atrial fibrillation. Circulation, 103(5):769-777. [doi:10.1161/01.CIR.103. 5.769]

Armour, J.A., Murphy, D.A., Yuan, B.X., et al., 1997. Gross and microscopic anatomy of the human intrinsic cardiac nervous system. Anat. Rec., 247(2):289-298. [doi:10. 1002/(SICI)1097-0185(199702)247:2<289::AID-AR15> 3.0.CO;2-L]

Chang, H.Y., Lo, L.W., Lin, Y.J., et al., 2013. Long-term outcome of catheter ablation in patients with atrial fibrillation originating from nonpulmonary vein ectopy. $J$. Cardiovasc. Electrophysiol., 24(3):250-258. [doi:10. 1111/jce.12036]

Chen, S.A., Tai, C.T., Yu, W.C., et al., 1999. Right atrial focal atrial fibrillation: electrophysiologic characteristics and radiofrequency catheter ablation. J. Cardiovasc. Electrophysiol., 10(3):328-335. [doi:10.1111/j.1540-8167. 1999.tb00679.x]

Ganesan, A.N., Shipp, N.J., Brooks, A.G., et al., 2013. Long-term outcomes of catheter ablation of atrial fibrillation: a systematic review and meta-analysis. J. Am. Heart Assoc., 2(2):e004549. [doi:10.1161/JAHA.112. 004549]

Goette, A., Honeycutt, C., Langberg, J.J., 1996. Electrical remodeling in atrial fibrillation. Time course and mechanisms. Circulation, 94(11):2968-2974. [doi:10.1161/01. CIR.94.11.2968]

Haïssaguerre, M., Jaïs, P., Shah, D.C., et al., 1998. Spontaneous initiation of atrial fibrillation by ectopic beats originating in the pulmonary veins. N. Engl. J. Med., 339(10):659-666. [doi:10.1056/NEJM199809033391003]

Jiang, R.H., Po, S.S., Tung, R., et al., 2014. Incidence of pulmonary vein conduction recovery in patients without clinical recurrence after ablation of paroxysmal atrial fibrillation: mechanistic implications. Heart Rhythm., 11(6): 969-976. [doi:10.1016/j.hrthm.2014.03.015]

Lerman, B.B., Belardinelli, L., 1991. Cardiac electrophysiology of adenosine. Basic and clinical concepts. Circulation, 83(5):1499-1509. [doi:10.1161/01.CIR.83.5.1499]

Lin, W.S., Tai, C.T., Hsieh, M.H., et al., 2003. Catheter ablation of paroxysmal atrial fibrillation initiated by non-pulmonary vein ectopy. Circulation, 107(25):31763183. [doi:10.1161/01.CIR.0000074206.52056.2D]

Morillo, C.A., Klein, G.J., Jones, D.L., et al., 1995. Chronic rapid atrial pacing. Structural, functional, and electrophysiological characteristics of a new model of sustained atrial fibrillation. Circulation, 91(5):1588-1595. [doi:10. 1161/01.CIR.91.5.1588]

Morita, H., Zipes, D.P., Morita, S.T., et al., 2012. The role of coronary sinus musculature in the induction of atrial fibrillation. Heart Rhythm., 9(4):581-589. [doi:10.1016/j. hrthm.2011.11.041]

Nakagawa, H., Scherlag, B.J., Patterson, E., et al., 2009. Pathophysiologic basis of autonomic ganglionated plexus ablation in patients with atrial fibrillation. Heart Rhythm., 6(12 Suppl.):S26-S34. [doi:10.1016/j.hrthm.2009.07.029]

Patterson, E., Po, S.S., Scherlag, B.J., et al., 2005. Triggered firing in pulmonary veins initiated by in vitro autonomic nerve stimulation. Heart Rhythm., 2(6):624-631. [doi:10. 1016/j.hrthm.2005.02.012]

Po, S.S., Scherlag, B.J., Yamanashi, W.S., et al., 2006. Experimental model for paroxysmal atrial fibrillation arising at the pulmonary vein-atrial junctions. Heart Rhythm., 3(2):201-208. [doi:10.1016/j.hrthm.2005.11.008]

Shah, D., Haïssaguerre, M., Jaïs, P., et al., 2003. Nonpulmonary vein foci: do they exist? Pacing Clin. Electrophysiol., 26(7 Pt 2):1631-1635. [doi:10.1046/j.1460-9592.2003.t011-00243.x]

Sotomi, Y., Inoue, K., Ito, N., et al., 2013. Cause of very late recurrence of atrial fibrillation or flutter after catheter ablation for atrial fibrillation. Am. J. Cardiol., 111(4): 552-556. [doi:10.1016/j.amjcard.2012.10.040]

Wijffels, M.C., Kirchhof, C.J., Dorland, R., et al., 1995. Atrial fibrillation begets atrial fibrillation. A study in awake chronically instrumented goats. Circulation, 92(7): 1954-1968. [doi:10.1161/01.CIR.92.7.1954]

Workman, A.J., Kane, K.A., Rankin, A.C., 1999. Ionic basis of a differential effect of adenosine on refractoriness in rabbit AV nodal and atrial isolated myocytes. Cardiovasc. Res., 43(4):974-984. [doi:10.1016/S0008-6363(99)00166-2]

Yamaguchi, T., Tsuchiya, T., Miyamoto, K., et al., 2010. Characterization of non-pulmonary vein foci with an EnSite array in patients with paroxysmal atrial fibrillation. 
Europace, 12(12):1698-1706. [doi:10.1093/europace/ euq326]

Zimmermann, M., Kalusche, D., 2001. Fluctuation in autonomic tone is a major determinant of sustained atrial arrhythmias in patients with focal ectopy originating from the pulmonary veins. J. Cardiovasc. Electrophysiol., 12(3):285-291. [doi:10.1046/j.1540-8167.2001.00285.x]

Zipes, D.P., Knope, R.F., 1972. Electrical properties of the thoracic veins. Am. J. Cardiol., 29(3):372-376. [doi:10. 1016/0002-9149(72)90533-4]

\section{中文㔍要:}

本文题目: 阵发性房颤患者肺静脉隔离前后非肺静脉触发灶的诱发情况及随访研究

Non-pulmonary vein foci induced before and after pulmonary vein isolation in patients undergoing ablation therapy for paroxysmal atrial fibrillation: incidence and clinical outcome

研究目的: 观察阵发性房颤患者分别在肺静脉隔离前后联合使用三磷酸腺苷 (ATP) +异丙 (去甲) 肾

上腺素（ISP）对非肺静脉触发灶的诱发情况以及消融非肺静脉触发灶后的临床疗效。

创新要点: 发现肺静脉电学隔离前肺静脉在触发房颤方面起主要作用, 环肺静脉隔离术显著降低了肺 静脉的放电情况, 却同时增强了肺静脉以外的部位触发房颤的能力。

研究方法: 选择从 2010 年 4 月至 2011 年 1 月在浙江大学附属邵逸夫医院首次行房颤射频消融术的药 物治疗无效, 发作时症状明显的 80 例阵发性房颤患者。所有的患者均于肺静脉隔离前后通 过静脉给予 ATP (20 mg) +ISP (20 $\mu \mathrm{g} / \mathrm{min})$ 诱发房颤。诱发出房颤的起始部分被标记和 记录（诱发过程详见图 2）。术后常规口服华法林抗凝不少于 3 个月，控制 INR 值 2 3; 术后 3 个月停用所有抗心律失常药物; 术后 1 个月、 3 个月、 6 个月、 1 年、 2 年时及当病人 有心悸等症状时通过 24 小时动态心电图对病人随访。

重要结论: ATP+ISP 可以有效诱发阵发性房颤患者的非肺静脉触发灶, 特别是在环肺静脉隔离以后。 术中对非肺静脉触发灶进行消融可能使病人获益, 虽然在本研究中随访结果没有达到统计 学上的显著差异。

关键词组: 阵发性房颤; 非肺静脉触发灶; 三磷酸腺苷 